\title{
Online API Controller for Performance Improvements in Multilevel STATCOMs
}

\author{
Ayman Alhejji \\ Department of Electrical and Electronics Engineering Technology, Yanbu Industrial College, \\ Yanbu Industrial City, 46452, Saudi Arabia \\ Corresponding Author \\ ORCID:0000-0003-3820-2655
}

\begin{abstract}
This paper investigates improvements in the safety and operating range of transmission lines by incorporating a static synchronous compensator (STATCOM). A multilevel STATCOM-based voltage source converter (VSC) is used to ensure the safety and continuity of delivered power to a grid and is applied for emitting reactive power and reducing voltage instability resulting from abnormal operation. The key contribution of this framework is an online adaptive proportional integral (API) controller applied to a STATCOMbased VSC control circuit to obtain rapid performance and voltage stability improvements for the generation system output. The system architecture is simulated in MATLAB/Simulink, including a generation integrated with an infinite bus and STATCOM-based VSC. The simulation indicated the voltage instability at the point of common coupling (PCC) effectively declined by approximately $8 \%$ using the API controller and declined by $10 \%$ for PI controller that is tuned by the grey wolf optimization (GWO) under a sudden load variation. The results corroborate the findings obtained for the API controllers, which are technically compared with those for the GWO-based PI controllers.
\end{abstract}

Keywords: API controller; Grey wolf optimization; STATCOM; Point of Common Coupling; Reactive power.

\section{INTRODUCTION}

Reactive power compensation control has recently gained importance in power systems due to its ability to decrease transmission losses, limit large amplitude variations in the receiving end voltages, and increase power transmission capabilities. Therefore, flexible alternative current transmission systems (FACTS) have been offered to the marketplace since the 1980 s in order to solve power quality issues related to the compensation of nonlinear loads, poor power factors and imbalanced loads [1]. These FACTS devices, such as static var compensators (SVCs), thyristorcontrolled reactors (TCRs), thyristor-switched reactors (TSRs), thyristor-switched capacitors (TSCs) and static synchronous compensators (STATCOMs), are implemented in parallel with the network. The most popular compensator of the FACTS devices used in distribution networks and transmission lines is the STATCOM device. There are two STATCOM topologies: the STATCOM-based current source converter topology and the STATCOM-based voltage source converter topology. The former includes a current source converter with a series reactor on the DC side or DC-link and shunt capacitor on the AC side; the latter essentially includes one voltage source converter (VSC) with a capacitor on the $\mathrm{DC}$ side/DC-link and a series reactor on the AC side or a coupling transformer on the AC grid side [2]. STATCOMbased VSC is preferred due to its self-protection from reverse voltage issues and high efficiency compared with STATCOM using a current source converter.

STATCOM is emulated by a variable voltage source, and it has the ability to generate and absorb reactive power with a fast response due to the high switching frequency and lack of moving parts [1-2]. Unlike the static var compensator (SVC), the modern STATCOM has a faster response, smaller size, wider operating range and lower harmonic current. Applying a STATCOM in power energy systems improves stability, increases the capability of power transfer and enhances power quality [3-4]. Due to the imbalance of the real power flows between wind generators and the grid, a wind generator could lose synchronization with electrical networks under abnormal operation; hence, a coordinated current control was proposed between a wind farm and STATCOM in [5]. A STATCOM has actively been utilized in grid-connected alternative energy applications due to its capability for enhancing system performance and mitigating renewable source integration into the grid [6]. Moreover, in [7], the coordination of the control strategy between the PV power system and STATCOM during grid disturbance was studied. In a distribution network, to deliver power with superb quality, distributed STATCOMs play a vital role in mitigating voltage fluctuations and promoting the voltage profile [8].

Various applications have used the STATCOM compensator. For example, a \pm 100 -MVAr STATCOM was applied to a $161-\mathrm{kV}$ distribution network to rapidly regulate the voltage level and avoid the occurrence of voltage collapse [9-10]. Due to the impact of wind fluctuations on the output wind power delivered to the grid, a STATCOM was suggested to enhance the stability of the frequency, voltage and power quality. In [11], a \pm 50 -MVAr STATCOM was implemented in a $220-\mathrm{kV}$ power substation to increase the overall system stability performance of the power transmission capacity in a distribution network. A multilevel STATCOM-based voltage source converter (VSC) is used in this paper. The main purpose of multilevel STATCOM applications was described in [4]. Subsequently, many studies of multilevel STATCOMs 
have been published in the literature [5]-[16] and noted the key benefits, such as curtailing the voltage variations in DClink capacitors, reducing switching losses and maintaining harmonic performance.

Numerous optimization techniques for adjusting proportionalintegral (PI) control parameters and promoting the behavior of STATCOM models have been proposed [12-17]. For instance, a cuckoo search optimization method was used to tune PI control parameters and optimally control STATCOM output [12]. In addition, a genetic algorithm was used to adjust the PI controller in the STATCOM control scheme in [13-14]. In [15], to tune the optimal parameters of the STATCOM controller with other power system components, three optimization techniques, namely, a genetic algorithm, a particle swarm algorithm and an imperialist competitive algorithm, were effectively compared. Additionally, as noted in [16], a simplex algorithm was developed to tune the PI controller in a control circuit of a STATCOM device. Furthermore, an improved artificial bee colony algorithm was applied to optimize the PI control parameters of the STATCOM model in [17].

Different advanced control techniques for STATCOM compensators [18-23] have been proposed to control the VAR flow between wind power energy and STATCOM. Thus, the voltage at the point of common coupling (PCC) can be improved to ensure power continuity. These different control techniques, such as STATCOM-based fuzzy PI control [18], STATCOM-based adaptive control using deep deterministic policy gradient algorithm along with neural network estimation [19], the input-output feedback linearization of nonlinear STATCOM [20], robust STATCOM-based active disturbance rejection [21] and balance control techniques for imbalanced DC capacitor voltages in multilevel STATCOM units [22], have successfully been implemented to improve the overall power system efficiency. Additionally, as noted in [23], heuristic dynamic programming was used to promote oscillation damping by coordinating a reactive power control strategy involving STATCOM and a wind farm during fault periods.

Also, all existing literature studies of STATCOM-based VSCs have not discussed adaptive controller to overcome the challenge of the excessive reactive power compensation into grid that could lead to increase power losses and decrease active power flow.

To overcome this challange, the paper presents a new approach for rapid and sufficient reactive power compensation in energy transmission. The proposed online and self-tuning API controllers typically rely on an adaptive mechanism. This adaptive mechanism is dedicated to updating unknown proportional and integral parameters that are determined by the actuating signal under present conditions. Then, the API controller is applied to a multilevel STATCOM-based VSC control circuit that can obtain a rapid and sufficient response and maintain voltage stability at the PCC bus based on the desired reference voltage. The system architecture, including power generation integrated with an infinite bus and a STATCOM-based VSC, is simulated in MATLAB/Simulink. The simulations validate that the voltage instability at the PCC controllably declined by approximately $8 \%$ using the API controller and by $10 \%$ for the PI controller that is tuned by the GWO all under load variations. The results corroborate the findings obtained for the API controller, which are compared with those for the GWO-based PI controller. Consequently, these API controllers behave in accordance with the present system environment, and adaptive STATCOM control could protect the system from resulting in cascading power outages.

\section{SYSTEM MODELING}

The cascade multilevel STATCOM model based on VSC in the $d-q$ reference frame is schematically depicted in Fig. 1.

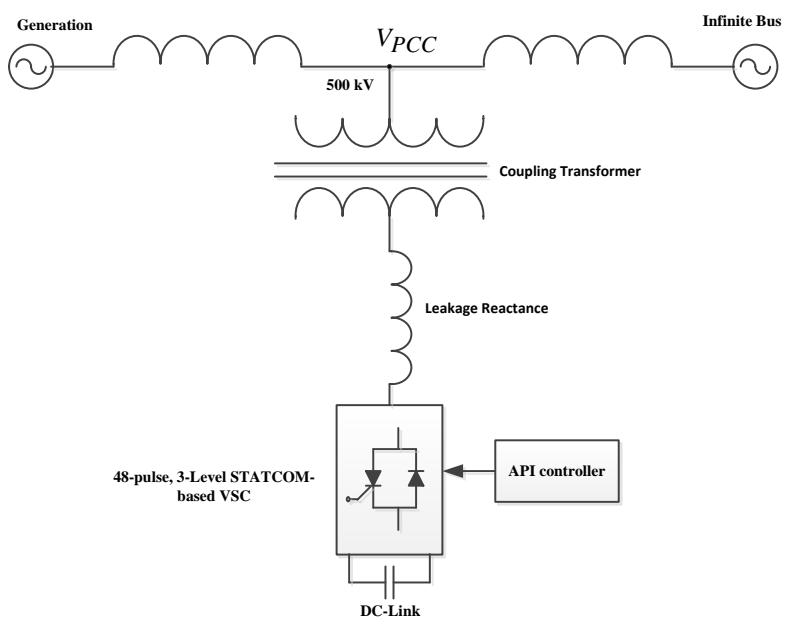

Fig. 1. Three-level, 48-pulse three-phase STATCOM topology

$$
\begin{aligned}
\frac{d_{i d}}{d t} & =-\frac{R}{L} i_{d}+\omega_{s} i_{q}-\frac{V_{d c}}{L} U_{1}+\frac{1}{L} v_{d} \\
\frac{d_{i q}}{d t} & =-\frac{R}{L} i_{q}-\omega_{s} i_{d}-\frac{V_{d c}}{L} U_{2}+\frac{1}{L} v_{q} \\
\frac{d V_{d c}}{d t} & =\frac{3}{2} \frac{\left(V_{d} i_{d}+V_{q} i_{q}\right)}{C_{e q} V_{d c}}
\end{aligned}
$$

where $i_{d}$ and $i_{q}$ denote the currents of the direct (d) and quadratic (q) axis component reference frames, respectively; $V_{d c}$ represents the DC link voltage of the equivalent capacitor in the STATCOM model; $v_{d}$ and $v_{q}$ are the $\mathrm{d}-\mathrm{q}$ axis components of the three-phase voltage terminals. Additionally, $\omega_{s}$ describes the supply frequency in hertz; $R, L$ and $C_{e q}$ denote the AC line resistance variables in ohms, equivalent interface Henry inductance, and DC-side Farad capacitance, respectively; $C_{e q}$ is the equivalent STATCOM capacitor. Furthermore, $U_{1}$ and $U_{2}$ are the STATCOM voltages of the $\mathrm{d}-\mathrm{q}$ axis components of the modulation index given by

$$
\begin{aligned}
& U_{1}=k(\alpha) V_{d c} \cos (\delta) \\
& U_{2}=k(\alpha) V_{d c} \sin (\delta)
\end{aligned}
$$

where $k(\alpha)$ and $\delta$ represent the modulation ratio of the peak voltage of the STATCOM unit to the DC voltage, $V_{d c}$, and the 
phase shift of the STATCOM voltage vector position among the d-q axis components, respectively.

The formulas of the active power $P$ in watts and reactive power $Q$ in var based on the $\mathrm{d}-\mathrm{q}$ axis components of the STATCOM model are given by

$$
\begin{aligned}
& P=V i_{d} \\
& Q=-V i_{q}
\end{aligned}
$$

Notably, the active and reactive power of the STATCOM, which are decoupled controls, depend on the d-axis current and q-axis current, respectively. Technically, $i_{d}$ and $i_{q}$ are the active and reactive currents, respectively.

\section{STATCOM CONTROL STRATEGY}

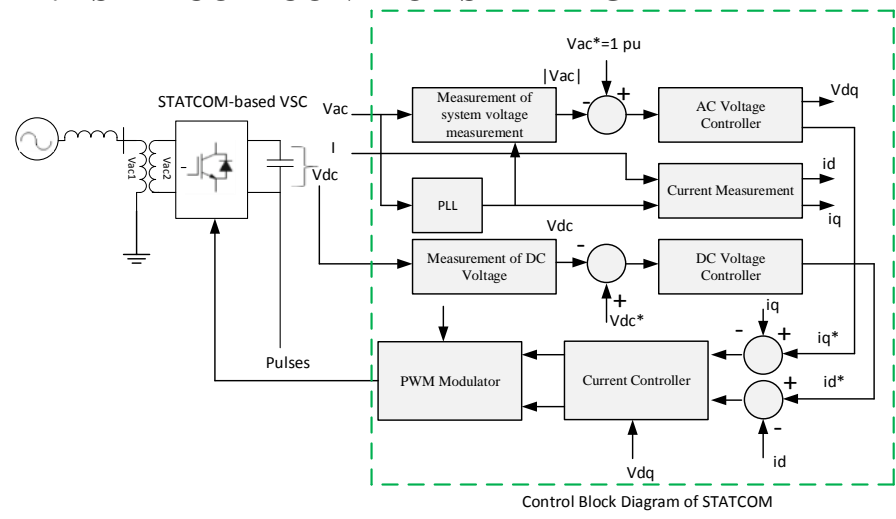

Fig. 2. Control of STATCOM-based VSCs

In this section, two control methods are presented: 1) PI controllers based on GWO and 2) API controllers. The STATCOM control scheme depends on the currents and voltages that are transformed into $\mathrm{d}-\mathrm{q}$ axis components. This STATCOM control scheme can be classified into two main tasks. The first task is to increase or decrease the DC-link capacitor voltage so that the generated $\mathrm{AC}$ voltage can be effectively controlled or has a correct amplitude for reactive power control. The second task of the STATCOM control scheme is to keep the AC-generated voltage of the STATCOM unit corresponding in phase with the system voltage, which is a PCC voltage. In other words, when the magnitude of the AC voltage $\left|V_{a c}\right|$ at the PCC becomes greater than the voltage reference $V_{a c}^{*}=1 p u$, the error signal is processed by the PI controller, generating a higher reactive current reference $i_{q}^{*}$ and more reactive current $i_{q}$. The current regulator/controller will then increase the angle $(\varphi)$ of the STATCOM converter voltage with respect to the voltage at the PCC so that the active power temporarily flows from the grid at the PCC bus to the DC-link capacitor. In fact, this process increases the DC voltage and consequently generates a higher voltage.

The AC voltage magnitude is represented as follows:

$$
\left|V_{a c}\right|=\sqrt{v_{d}^{2}+v_{q}^{2}}
$$

This is illustrated in Fig.2; the control scheme of the STATCOM system only involves measuring the three-phase voltages and currents at the PCC bus, and no direct reactive power measurements are needed. The three-phase voltages and currents are transformed into a d-q axis synchronous reference frame. Furthermore, to actuate the error signal, in the sense that the actual voltages and currents accurately follow the reference values, the actual $d-q$ axis components of voltages and currents are compared with their respective reference values. The error signal of the $\mathrm{AC}$ voltage regulator represents the difference between the voltage reference $V_{a c}^{*}$ and the magnitude of the voltage $\left|V_{a c}\right|$. Such a signal is controlled by the API controller generating the desired $i_{q}^{*}$, as shown in Fig. 2. Subsequently, another error signal between the current reference $i_{d}^{*}$ and actual value $i_{d}$ is driven by the API controller, which accurately determines the angle $\varphi$, as shown in Fig. 2. Then, this controlled angle $\varphi$ is combined with the phase angle $\theta$ of the grid voltage at the PCC. Moreover, the phase-locked loop is important in gridconnected systems such as STATCOM-based VSCs. The output of the PLL, which is a phase angle, is used to detect and determine the $\mathrm{d}-\mathrm{q}$ axis components of the three-phase voltages and currents synchronized in phase with the fundamental components of the STATCOM. This phase angle together with the controlled angle $\varphi$ can provide a sinusoid pulse width modulation (PWM), which is used to produce 48 pulses for STATCOM-based VSCs.

The reactive power flow between a STATCOM-based VSC and a PCC is regulated by the phase angle that represents the difference between the voltage PCC and the STATCOM voltage output, as shown in Fig. 1. Additionally, considering the balanced DC voltage used for energy storage, the DC voltage in STATCOM-based VSCs should be sufficiently large. That large DC voltage helps provide voltage amplitude matching based on the AC voltage in the secondary windings of the coupling transformer [10]. The control scheme is designed to realize the voltage difference which is error signal between the reference DC voltage and the actual DC voltage. Such an error signal is used by the API controller to generate an active current reference $i_{d}^{*}$ and promptly control the rate of active power exchange.

The control scheme of 3-level STATCOM-based VSCs consists of three regulators. For example, the outer voltage control loop (AC voltage regulator), inner current control (current regulator) and DC voltage control are the main control schemes of STATCOM units. The objective of the STATCOM control approach is to curtail or eliminate overvoltage issues under minor load conditions while regulating and maintaining the PCC bus voltage level under major load conditions. The flow rate of the reactive power exchange is actively determined based on the major difference in voltage amplitudes between STATCOM-based SVCs and the voltage PCC.

The development strategy of the 3-level STATCOM control scheme is presented. As shown in Fig 5, AC voltage regulation includes the voltage reference $V_{a c}^{*}$ as $1 p u$ and the magnitude of the AC voltage $\left|V_{a c}\right|$ as the actual signal. The error signal between the voltage reference and magnitude of the AC voltage is processed by the proposed PI controller to generate the desired $i_{q}^{*}$ reference. However, in the current control scheme, the PI controller handles the error signal of the reactive current reference $i_{q}^{*}$ and the actual reactive current $i_{q}$ to generate the desired voltage angle, which are combined to adjust the synchronized phase angle. This combination is used to produce sinusoidal pulse width modulation (SPWM) and generate 48 pulses for a 3-level STATCOM that employs a voltage source converter. The phase angle, which is 
synchronized in phase with the system voltage, can be used to determine the control strategy for a STATCOM-based SVC.

\section{III.I PI controller-based grey wolf optimization (GWO-PI)}

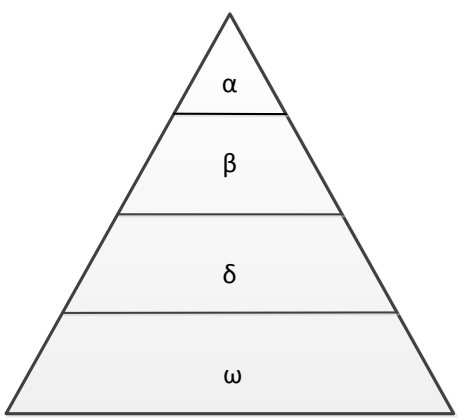

Fig. 3. Grey wolf leadership pyramid

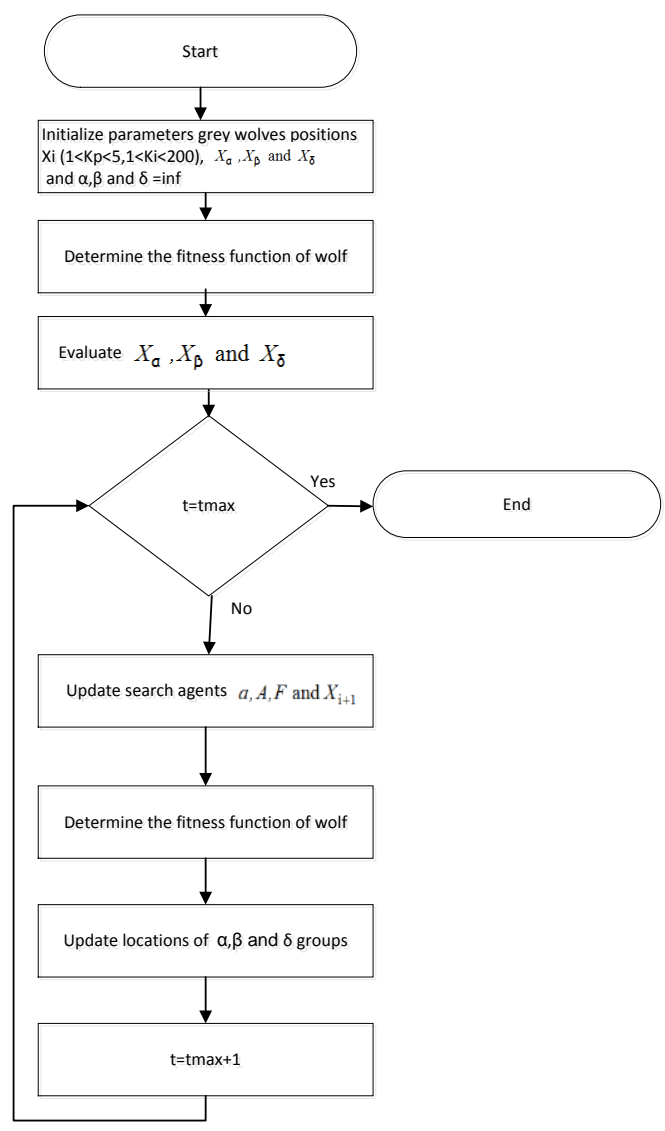

Fig. 4. Flowchart of the PI-based GWO algorithm

The grey wolf optimization (GWO) algorithm, which was proposed by Mirjalili, S. et al in 2014 [24], aims to perform the hierarchy of wolves' leadership. This GWO technique provides satisfactory accomplishment compared with other optimization techniques, such as the gravitational search, evolutionary programming, particle swarm optimization and differential evolution algorithms, as discussed in [24-27]. The GWO technique is well organized, and individuals are classified into four groups for hunting: $\alpha, \beta, \delta$ and $\omega$. By modeling the hierarchy of the grey wolves as an optimization process, the fittest solution is the leader named $\alpha$, followed by $\beta$ and $\delta$ which reflect the best solutions. Additionally, $\omega$ eventually encompasses the remaining solutions. This grey wolf hunting strategy algorithm mainly depends on several steps, such as chasing, hunting, and tracking or searching for prey, known as exploration, encircling prey, and attacking prey (or exploitation), based on a social hierarchy, which can be modeled as encircling prey at a specific position

$$
\begin{aligned}
\vec{E} & =\left|\vec{F} \cdot \vec{x}_{p i}-\vec{x}_{i}\right| \\
\vec{x}_{i+1} & =\vec{x}_{p i}-\vec{A} \cdot \vec{E}
\end{aligned}
$$

where $\vec{x}_{i}$ denotes the grey wolf location, $\vec{x}_{p i}$ is the food or prey, and $\vec{E}$ is the space between the grey wolf and the food. Moreover, $\vec{A}$ and $\vec{F}$ are vector parameters determined as follows:

$$
\begin{aligned}
& \vec{a}=2-2 \times t / m \\
& \vec{A}=2 \vec{a} \cdot \overrightarrow{r_{1}}-\vec{a} \\
& \vec{F}=2 \cdot \overrightarrow{r_{2}}
\end{aligned}
$$

where the $\vec{a}$ factor linearly and iteratively decays from 2 to $0, t$ is the time and $m$ denotes the maximum iteration value; both $\overrightarrow{r_{1}}$ and $\overrightarrow{r_{2}}$ describe the random vectors as $[0,1]$.

Two main principles are followed by grey wolves: search for prey (exploration) and attack prey (exploitation). First, looking for food is an exploration step that occurs by separating the search entities when $|A|>1$, and exploitation involves attacking prey when the search entities join together; in this case, $|A|<1$. The subsequent mathematical equations that describe the hunting positions and how other groups of $\beta$ and $\delta$ can provide support are given in (10)-(12).

$$
\left.\begin{array}{rl}
\vec{E}_{\alpha} & =\left|\vec{F} \cdot \vec{x}_{\alpha}-\vec{x}\right| \\
\vec{E}_{\beta} & =\left|\vec{F} \cdot \vec{x}_{\beta}-\vec{x}\right| \\
\vec{E}_{\delta} & =\left|\vec{F} \cdot \vec{x}_{\delta}-\vec{x}\right|
\end{array}\right\}
$$

The initial control parameters or constants of the PI controllers are selected as follows.

For the AC voltage controller,

For the current controller,

$$
\begin{gathered}
0.01 \leq k_{p v} \leq 20 \\
2800 \leq k_{\text {iv }} \leq 3200
\end{gathered}
$$

$$
\begin{aligned}
& 0.01 \leq k_{p i} \leq 10 \\
& 0.01 \leq k_{i i} \leq 50
\end{aligned}
$$

For the DC voltage controller,

$$
\begin{gathered}
5 \leq k_{p d c} \leq 40 \\
12 \leq k_{i d c} \leq 3000
\end{gathered}
$$

The results of the PI control parameters are obtained by the GWO technique, as shown in Table 1. 


\section{III.II Adaptive proportional integral (API) controller}

In the STATCOM control scheme, API controllers that provide online self-tuning are proposed to replace three conventional PI controllers for better performance. Such controllers could improve the regulation of the voltage profile at the PCC and reduce voltage instability in periods of rapid response. An AC voltage regulation includes the voltage reference $V_{a c}^{*}$ as $1 \mathrm{pu}$, the magnitude of the actual AC voltage $\left|V_{a c}\right|$, and the API controller. The error signal between $V_{a c}^{*}$ and $\left|V_{a c}\right|$ is processed by the API controller to rapidly generate the desired $i_{q}^{*}$ reference. However, in the current control scheme, the API controller for online self-tuning handles the error signal between the reactive current reference $i_{q}^{*}$ and the actual reactive current $i_{q}$, hence generating the desired voltage angle; this desired voltage angle is combined to enhance the synchronized phase angle. This combination produces sinusoidal pulse width modulation (SPWM) and generates pulses in the cascaded three-level STATCOM.

Consider Fig.5, where the error signal between the voltage reference and the actual voltage plus droop signal is given to identify adaptive laws/mechanisms for updating unknown proportional and integral control parameters in the online selftuning technique. Thereafter, the updated API controller directly uses the error signal to generate the desired reactive current reference.

The proposed control structure of all API controllers is given by

$$
\text { API controller }=\widehat{k}_{p} e+\widehat{k}_{i} \int_{0}^{t} e d t
$$

where $e$ expresses an error signal/actuating signal, and $\hat{k}_{p}$ and $\hat{k}_{i}$ are proportional and integral parameters, respectively, which are updated online based on the subsequent adaptive laws (13). These laws, which are used to adaptively tune the API parameters (12), technically encompass a continuous error signal during the online adaptation process, as given by

$$
\left.\begin{array}{c}
\hat{\hat{k}}_{p}=\mu e x_{u} \\
\hat{\hat{k}}_{i v}=\mu \int e x_{u}
\end{array}\right\}
$$

where $x_{u}$ describes an actual signal, such as $\left|V_{a c}\right|, i_{d}$ or $i_{q}$, according to the control scheme; $\mu$ is a positive constant that is regarded as an adaptation gain.

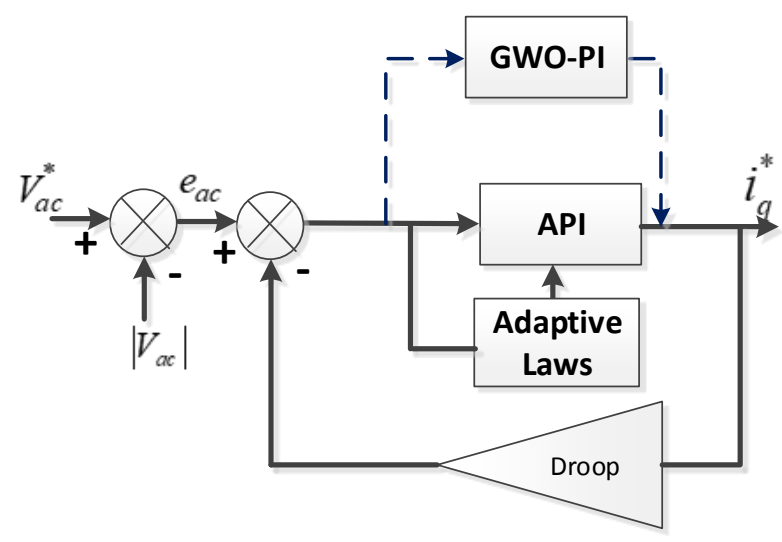

Fig. 5. AC voltage control diagram

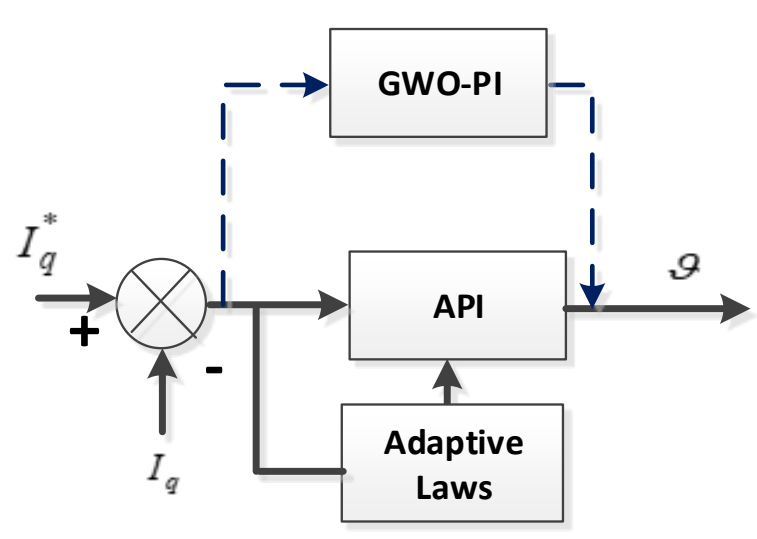

Fig. 6. AC current control diagram

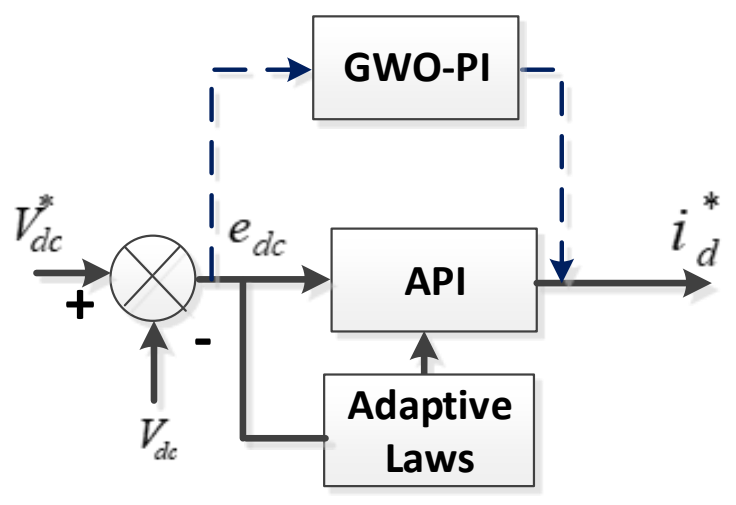

Fig. 7. DC voltage control diagram

\section{SIMULATION RESULTS}

For validity, the results of the proposed API controllers for a 3-level STATCOM are compared with those for tuned PI controllers utilizing the GWO technique. In Fig.5, the AC voltage reference at the PCC is 1 per unit $(p u)$, and the voltage droop is set to $\pm 3 \%$ for voltage modifications to support or withdraw (absorb) the reactive power to or from the grid. Similarly, the current voltage regulator for generating the desired angle $\varphi$ is dependent on the error signal of that generated reactive current reference $i_{q}^{*}$ from the AC voltage regulator and the actual reactive current $i_{q}$ so that the error signal can be effectively processed by the API controller.

A 100-Mvar, three-level, 48-pulse STATCOM-based VSC is used to control the PCC voltage at $500 \mathrm{kV}$. This VSC is principally connected to secondary windings of the coupling transformer, and primary windings are connected to the PCC. This STATCOM maintains a VSC voltage in phase with the grid voltage at the PCC during normal operation.

The following results illustrate how the total reactive power is mainly influenced by the VSC voltage and transformer leakage reactance during load variations. Furthermore, the majority of the VSC voltage is directly related to the DC voltage of the STATCOM; thus, the desired angle of the STATCOM-based VSC usually remains near zero and is momentarily phase shifted. In other words, the VSC voltage lag or lead could control the flow rate of active power caused by increasing or decreasing the DC voltage. 
Table 1. Tuned PI controllers through grey wolf optimization

\begin{tabular}{|c|c|c|c|}
\cline { 2 - 4 } \multicolumn{1}{c|}{} & $\begin{array}{c}\text { AC voltage } \\
\text { controller }\end{array}$ & $\begin{array}{c}\text { current } \\
\text { controller }\end{array}$ & $\begin{array}{c}\text { DC voltage } \\
\text { controller }\end{array}$ \\
\hline$k_{p}$ & 0.01 & 10 & 38 \\
\hline$k_{i}$ & 3200 & 35.775451 & 2950 \\
\hline
\end{tabular}

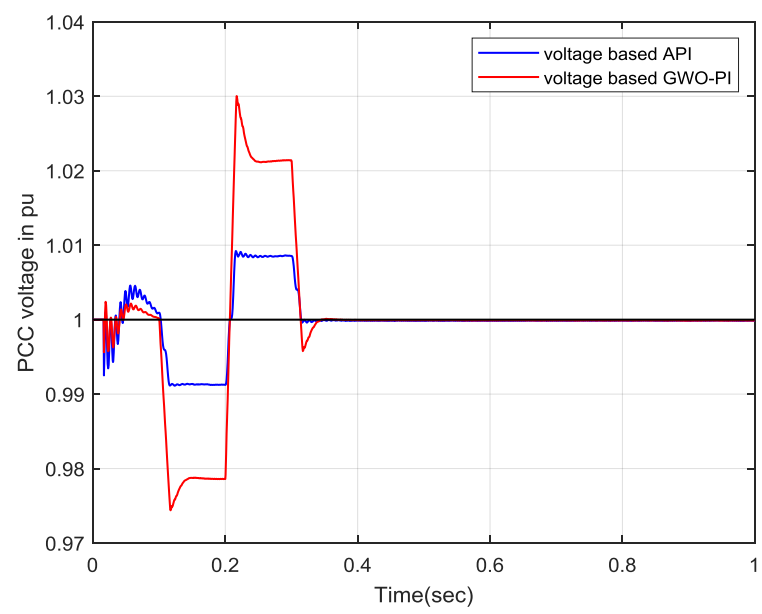

Fig. 8. PCC voltage in $p u$ for the API controller versus the GWO-PI controller.

Table 2. PCC voltage performance for the API controller versus the GWO-PI controller.

\begin{tabular}{|c|c|c|}
\cline { 2 - 3 } \multicolumn{1}{c|}{} & $\begin{array}{c}\text { Overshoot } \\
\text { (percentage })\end{array}$ & $\begin{array}{c}\text { Settling time } \\
(\mathrm{sec})\end{array}$ \\
\hline API controller & $0.9 \%$ & $0.3 \mathrm{sec}$ \\
\hline $\begin{array}{c}\text { GWO-PI } \\
\text { controller }\end{array}$ & $3 \%$ & $0.377 \mathrm{sec}$ \\
\hline
\end{tabular}

In Fig 8, the voltage profile results at the PCC bus were compared between the GWO-PI controller and API controller; both controllers yielded satisfactory performance and recovered the voltage PCC to the reference value of $1 \mathrm{pu}$. However, as shown in Table 2, at $0.22 \mathrm{sec}$, the result obtained by the API controller showed superior performance to that of the GWO-PI controller; notably, the voltage profile at the PCC reached $1.0098 \mathrm{pu}$ and was less affected by disturbances using the API controller, and the transient response lasted almost $0.3 \mathrm{sec}$. Additionally, for the GWO-PI controller, the PCC voltage reached approximately $1.03 p u$ at $0.22 \mathrm{sec}$, and even the transient response lasted almost $0.325 \mathrm{sec}$ before reaching a steady-state at $1 \mathrm{pu}$ as a reference value.

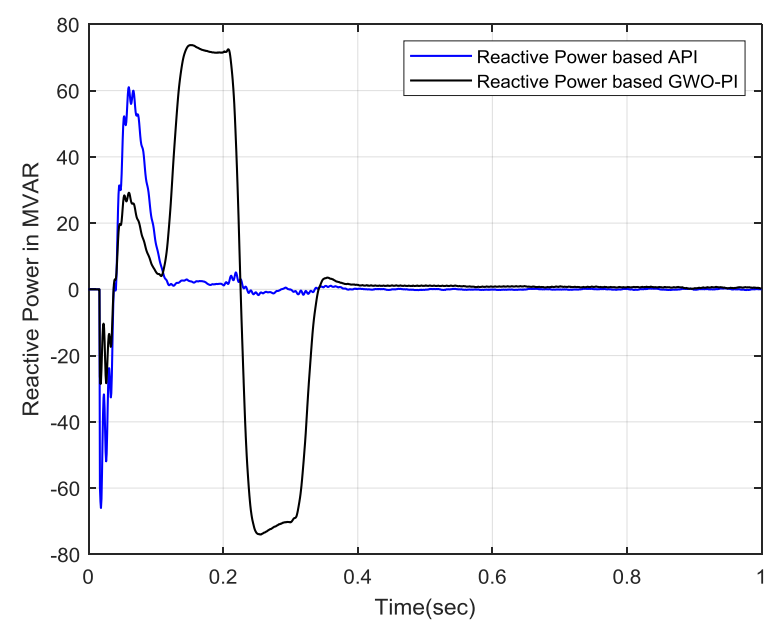

Fig. 9. Reactive power exchange of the 3-level STATOMbased SVC for different control methods.

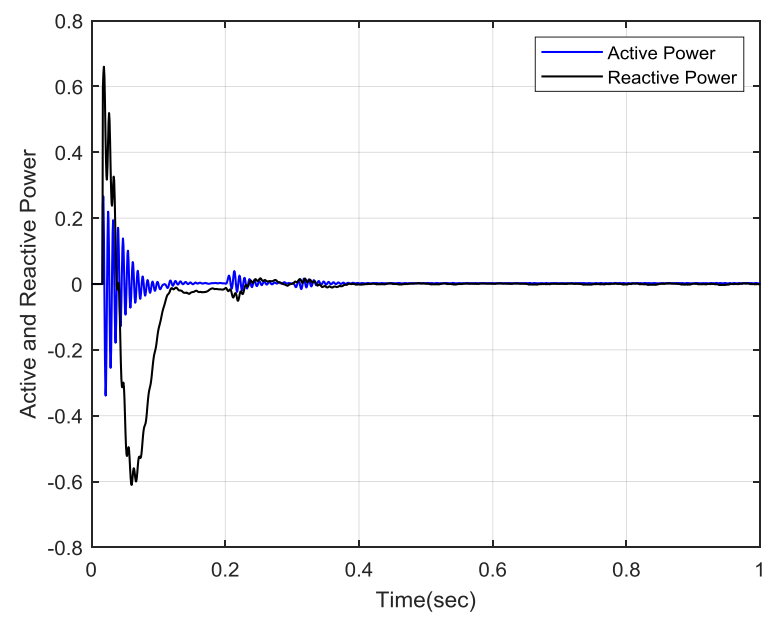

Fig. 10. PQ performance for the API controller.

Figure 9 displays the overall performance of the GWO-PI and API controllers, which were used in STATCOM to inject reactive power during load variation/grid fault. In normal operation, the STATCOM unit simply maintains VSC voltage synchronization with the voltage at the PCC.

The API controller outperformed the GWO-PI controller in providing reactive power compensation (+60 Mvar), which provided sufficient reactive power for the grid (+72 Mvar). When the grid fault occurred at $0.05 \mathrm{sec}$, the reactive power was rapidly injected into the grid by STATCOM based on the API controller to ensure the continuity of delivered power. In Fig. 10, the rapid variation in reactive power injection during the disturbance from $0.05 \mathrm{sec}$ to $0.2 \mathrm{sec}$ was successfully handled by the API controller. 


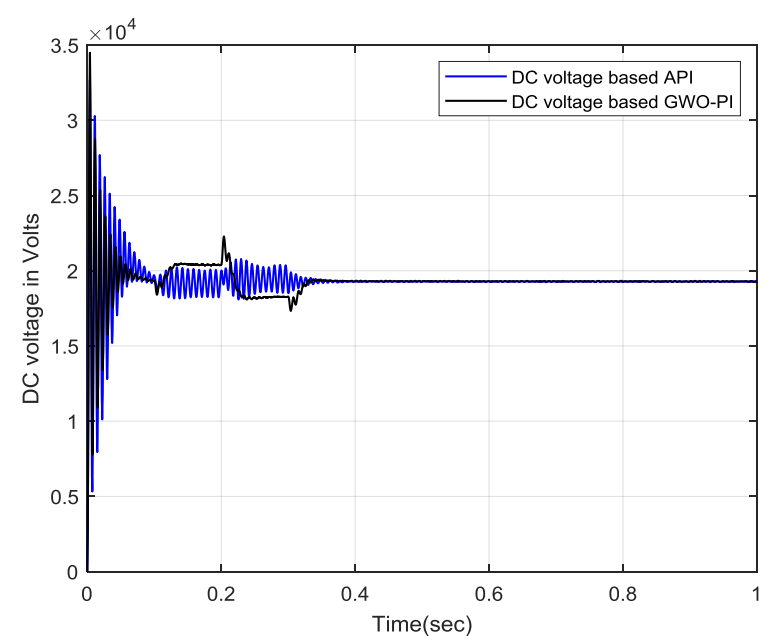

Fig. 11. DC-link voltage performance of the STATCOM in different control methods.

The results in Fig. 11 reflect the performance of the DC side of the STATCOM with the GWO-PI controller and API controller. The DC voltage of the STATCOM is $19.2 \mathrm{kV}$; however, the large overshoot initially reaches $30.4 \mathrm{kV}$ when using the GWO-PI controller, but this value only reaches $30.09 \mathrm{kV}$ using the proposed API controller. This result highlights the superiority of the API controller over the GWOPI controller.

\section{V.CONCLUSION}

An API controller was proposed to enhance transmission during abnormal operations in which the operating point of a multilevel STATCOM promptly varies; therefore, an API control strategy was applied to a 3-level STATCOM device to ensure the stable, fast and sufficient reactive power compensation at the PCC bus during any sudden changes in transmission and the continuity of delivered power to the grid. To ensure the validity of the proposed API method, a STATCOM with an API controller was compared to a STATCOM with a GWO-PI controller during sudden load variations. Both controllers displayed superb dynamic stabilities, but superior results were obtained by the API controller.

\section{REFERENCES}

[1] Enrique Acha , Claudio R. Fuerte-Esquivel, Hugo Ambriz-Pérez , César Angeles-Camacho , "FACTS Modeling and Simulation in Power Networks," 2004. ISBN-13: 978-0470852712.

[2] J. Jung, J. Lee, S. Sul, G. T. Son and Y. Chung, "DC Capacitor Voltage Balancing Control for DeltaConnected Cascaded H-Bridge STATCOM Considering Unbalanced Grid and Load Conditions,'IEEE Transactions on Power Electronics, vol. 33, no. 6, pp. 4726-4735, June 2018.doi: 10.1109/TPEL.2017.2730244

[3] Hale Bakir, Ahmet Afsin Kulaksiz , "Modelling and voltage control of the solar-wind hybrid micro-grid with optimized STATCOM using GA and BFA," Engineering Science and Technology, an International Journal, Augest 2019. https://doi.org/10.1016/j.jestch.2019.07.009.

[4] A. Halder, D. Mondal and N. Pal, "Nonlinear Optimal STATCOM Controller for Power System Based on Hamiltonian Formalism," 2018 IEEE International Conference on Power Electronics, Drives and Energy Systems (PEDES), Chennai, India, 2018, pp. 1-6. doi: 10.1109/PEDES.2018.8707486.

[5] H. Geng, L. Liu and R. Li, "Synchronization and Reactive Current Support of PMSG-Based Wind Farm During Severe Grid Fault," IEEE Transactions on Sustainable Energy, vol. 9, no. 4, pp. 1596-1604, Oct. 2018. doi: 10.1109/TSTE.2018.2799197

[6] Mohod, S.W., Aware, M.V.,"A STATCOM-control scheme for grid connected wind energy system for power quality improvement," IEEE Syst. J., vol 4 , no.3, pp. 346-352, June 2010.

[7] Yang,L.,Liu,W.,Peng,G.,Chen,Y.-

G.,Xu,Z., "Coordinated-control strategy of photovoltaic converters and static synchronous compensators for power system fault ride-through," Electr.PowerCompon.Syst., vol 44, pp.1683-1692, August 2016.

[8] Reza Sirjania, Ahmad Rezaee Jordehib , “Optimal placement and sizing of distribution static compensator (D-STATCOM) in electric distribution networks: A review," Renewable and Sustainable Energy Reviews, vol 77,pp. 688-694, September 2017.

[9] C. Schauder, M. Gernhardt, E. Stacey, T. Lemak ,L. Gyugyi ,T.W. Cease and A. Edris, “Operation of /spl plusmn/100 MVAr TVA STATCON," IEEE Transactions on Power Delivery, vol. 12, no. 4, pp. 1805-1811, Oct. 1997. doi: 10.1109/61.634209

[10] B. Singh, R. Saha, A. Chandra and K. AlHaddad,"Static synchronous compensators (STATCOM): a review,"IET Power Electronics, vol. 2, no. 4, pp. 297-324, July 2009.

[11] Q. Song, W. Liu and Z. Yuan ,"Multilevel Optimal Modulation and Dynamic Control Strategies for STATCOMs Using Cascaded Multilevel Inverters,'IEEE Transactions on Power Delivery, vol. 22, no. 3, pp. 1937-1946, July 2007. doi: 10.1109/TPWRD.2007.899771.

[12] N. Nayak, B. Rath and P. K. Rout ,"Dynamic Stability Improvement of PSS and STATCOM Based Power System Using Cuckoo Search Based PI Controller,'2nd International Conference on Computational Intelligence and Networks (CINE), Bhubaneswar, 2016, pp. 139144. doi: 10.1109/CINE.2016.31

[13] A. Z. Hussein, N. H. Saad and S. F. Mekamer,"Reactive Power and Voltage Control of Offshore Wind Parks Based PI Controller Tuning for STATCOM via Genetic Algorithm," Twentieth International Middle East Power Systems Conference (MEPCON), Cairo, Egypt, 2018, pp. 558-563. doi: 10.1109/MEPCON.2018.8635275. 
[14] H. Bakir and A. A. Kulaksiz,"Modelling and Voltage Control of the Solar-Wind Hybrid Micro-Grid with Optimized STATCOM,’23rd International Conference Electronics, Palanga, Lithuania, 2019, pp. 1-6.doi: 10.1109/ELECTRONICS.2019.8765575.

[15] M. J. Morshed and A. Fekih,“A Probabilistic Robust Coordinated Approach to Stabilize Power Oscillations in DFIG-Based Power Systems," IEEE Transactions on Industrial Informatics, vol. 15, no. 10, pp. 5599-5612, Oct. 2019.doi: 10.1109/TII.2019.2901935.

[16] A. M. Vural and K. C. Bayindir, "Optimization of parameter set for STATCOM control system," IEEE PES T\&D 2010, New Orleans, LA, 2010, pp. 1-6.doi: 10.1109/TDC.2010.5484230

[17] M. Wang, L. Wang, X. Chang and X. Wang, "An Original Self-Turning PI Controller for STATCOM in Wind Farm with Voltage Fluctuation Based on IABC Algorithm," IEEE 10th International Symposium on Power Electronics for Distributed Generation Systems (PEDG), Xi'an, China, 2019, pp. 395-400. doi: 10.1109/PEDG.2019.8807658

[18] A. Luo, C. Tang, Z. Shuai, J. Tang, X. Y. Xu and D. Chen, "Fuzzy-PI-Based Direct-Output-Voltage Control Strategy for the STATCOM Used in Utility Distribution Systems," in IEEE Transactions on Industrial Electronics, vol. 56, no. 7, pp. 2401-2411, July 2009. doi: 10.1109/TIE.2009.2021172.

[19] Zhanga, G., Hua,W., Caoa,D., Yia,J, Huanga,Q., Liub,Z., Chenb,Z., Blaabjergb, F., " A data-driven approach for designing STATCOM additional damping controller for wind farms," International Journal of Electrical Power \& Energy Systems, vol 117, pp. 105620, May 2020.

[20] D. Soto and R. Pena, "Nonlinear control strategies for cascaded multilevel STATCOMs," in IEEE Transactions on Power Delivery, vol. 19, no. 4, pp.
1919-1927, Oct. doi: 10.1109/TPWRD.2004.835394.

[21] M. Wang, X. Wang, J. Qiao and L. Wang, "Improved Current Decoupling Method for Robustness Improvement of LCL-type STATCOM Based on Active Disturbance Rejection Control," IEEE Access, vol. 7, pp. 121781-121792, July 2019.

[22] Z. Liu, B. Liu, S. Duan and Y. Kang, “A Novel DC Capacitor Voltage Balance Control Method for Cascade Multilevel STATCOM," IEEE Transactions on Power Electronics, vol. 27, no. 1, pp. 14-27, Jan. 2012. doi: 10.1109/TPEL.2010.2050337.

[23] W. Qiao, R. G. Harley and G. K. Venayagamoorthy, "Coordinated Reactive Power Control of a Large Wind Farm and a STATCOM Using Heuristic Dynamic Programming,"IEEE Transactions on Energy Conversion, vol. 24, no. 2, pp. 493-503, June 2009.

[24] S. Mirjalili, S. M. Mirjalili, and A. Lewis, "Grey wolf optimizer," Adv.Eng. Softw., vol. 69, pp. 46-61, Mar. 2014.

[25] Song, X. ,Tang, L. , Zhao, S. , Zhang, X. , Li, L., Huang, J. and Cai, W., "Grey Wolf optimizer for parameter estimation in surface waves," Soil Dyn. Earthq. Eng., vol 75, pp. 147-157, August 2015.

[26] Sulaiman, M. H. , Mustaffa, Z. , Mohamed, M. R. and Aliman, O., "Using the Grey Wolf optimizer for solving optimal reactive power dispatch problem," Appl. Soft Comput, vol 32, pp. 286-292, July 2015.

[27] Mahdad, B., Srairi, K., "Blackout risk prevention in a smart grid based flexible optimal strategy using Grey Wolf-pattern search algorithms," Energy Convers. Manage., 2015, 98, pp. 411-429. 